\title{
Impactos en la salud de la contaminación del aire
}

\author{
Health impacts of air pollution
}

Desde hace muchos años la Sociedad Chilena de Enfermedades Respiratorias (SER) ha estado preocupada de la Contaminación del aire (CA) través de su Comisión 'ad hoc'la que agrupa a miembros de la SER interesados por este tema de Salud Pública. La comisión ha mantenido a la SER y a los medios de comunicación actualizados en el tema, organizando periódicamente simposios en actividades cientificas de la Sociedad.

La Jornada de Otoño de 1990 abordó los efectos de la CA intra y extradomiciliaria en la salud y en 1991 un número completo de la Revista Chilena de Enfermedades Respiratorias fue dedicado a este tema que en esa época se focalizaba principalmente en Santiago ${ }^{1}$. Las jornadas de Otoño del 21 y 22 de mayo del presente año tituladas "Impacto en la salud de la Contaminación del aire", constituyen la segunda oportunidad en 39 años de existencia de esta actividad de la SER dedicada a tratar el impacto de la CA en la salud.

Saludamos esta iniciativa del directorio de la SER liderado por su presidenta Dra. Laura Mendoza y que fue impecablemente ejecutada por los coordinadores de las Jornadas, los Dres. Mauricio Césped y Guillermo Zepeda en sus aspectos de Medicina Respiratoria y Pediatría Neumológica respectivamente. En esta editorial presentamos una síntesis de los aspectos que consideramos más destacables de las exposiciones realizadas en estas jornadas.

Las variaciones climáticas tienen efectos en la salud humana, reportándose exceso de mortalidad asociados a ondas de calor. En Chile se constata un equilibrio entre el exceso de muertes por temperaturas extremas (muertes por frío versus muertes por calor). En estudios epidemiológicos se ha observado que frente al exceso de muertes por aumento de material particulado de $10\left(P M_{10}\right)$ y 2,5 $\left(P M_{2,5}\right)$, los aumentos en la humedad relativa resultan protectores. Aumentos de $1 \mathrm{mg} / \mathrm{m}^{3}$ en niveles de monóxido de carbono (CO) se asocian con riesgo en poblaciones vulnerables (edades extremas, embarazadas, pacientes cardiovasculares y respiratorios; comunidades con mayor densidad poblacional o distantes de la costa; deficiente arborización; e inequidad médica por índice de Gini). El impacto de la exposición a extremos térmicos, produce daño individual, poblacional y laboral y requieren de acciones preventivas. Los países más vulnerables a este efecto son aquellos que presentan mayor $C A^{2}$.

En un estudio realizado en 652 ciudades incluyendo 4 ciudades de Chile, se encontró que un aumento de $10 \mu \mathrm{g} / \mathrm{m}^{3}$ en el promedio móvil de 2 dias de la concentración de PM $M_{10}$ (promedio sobre el día actual y el anterior), se asoció con aumentos del 0,44\% en mortalidad diaria por todas las causas, $0,36 \%$ en mortalidad cardiovascular diaria, y 0,47\% en mortalidad respiratoria ${ }^{3}$.

Las enfermedades cardiovasculares y respiratorias concentran la mayor parte de la mortalidad atribuible a la CA. Modelos de riesgo ajustados para individuos, hogares y factores geográficos ${ }^{4}$, sugieren que aumentos de $10 \mu \mathrm{g} / \mathrm{m}^{3}$ de $P M_{2.5}$ se asocian con mayor riesgo de enfermedades cardiovasculares, accidente cerebrovascular y mortalidad por enfermedad cardiovascular. Este exceso de riesgo, aunque discreto, es relevante dada la alta proporción de población expuesta. Concentraciones elevadas de $P M_{2,5}$ se asocian al largo plazo con mayor riesgo de enfermedad cardiovascular en adultos de 35 a 70 años. La CA es un importante factor global de riesgo de enfermedades cardiovasculares siendo necesario reducir los niveles de contaminación, especialmente en países de ingresos 'per cápita' medios y bajos, donde los niveles de contaminación son más altos ${ }^{4}$.

El sistema inmune y el sistema mucociliar transportador desempeña un importante papel en la protección de las vías aéreas que diariamente filtran $9 \mathrm{~m}^{3}$ de aire con diversos grados de concentración de variados contaminantes. 
La exposición a contaminantes ambientales puede modificar el microbioma humano hecho que abre una prometedora veta de investigación. La contaminación por material particulado $P M_{10}$ y $P M_{2,5}$ determina aumento en la frecuencia de infecciones virales infantiles (influenza, VRS, resfríos) y en escolares incrementa la tasa de hospitalización por influenza. Ciudades con más de 100 días de CA elevada, muestran mayores tasas de infección por COVID-19.

Se ha descrito disminución de la función pulmonar, tanto en volumen como en flujos, en niños expuestos a la contaminación del aire. Aunque la magnitud del efecto es funcionalmente marginal en términos absolutos (10 a $16 \mathrm{ml} /$ año en $C V F$ y $\left.V E F_{1}\right)$, sus efectos a largo plazo pueden ser relevantes. Independientemente de la acción posterior de otros factores en la función pulmonar, la exposición temprana a CA expone a la población pediátrica a presentar menores valores basales de función pulmonar. Así, en el caso de desarrollar patología como una enfermedad pulmonar obstructiva crónica (EPOC), estos futuros adultos enfrentarán esta enfermedad con menores valores de función pulmonar que la población no expuesta tempranamente a $C A^{5}$.

Entre los mecanismos involucrados en el desarrollo del asma inducida o exacerbada por la CA se han descrito el daño por estrés oxidativo sostenido, efectos derivados de la remodelación de la vía aérea, inflamación y mayor sensibilización a aeroalergenos. Se estima globalmente que 13\% de nuevos asmáticos pueden ser atribuidos a la exposición a dióxido de nitrógeno $\left(\mathrm{NO}_{2}\right)$ y sus derivados, cifra que alcanzaría al 30\% en base a antecedentes del monitoreo de la calidad del aire en la ciudad de Santiago ${ }^{6}$.

Las concentraciones de $\mathrm{CO}$ y $\mathrm{NO}_{2}$ se asocian en forma directa con episodios de Infecciones respiratorias agudas. Las especies derivadas del óxido nitroso (NOx) muestran asociación positiva con episodios de bronquiolitis. Estudios en la ciudad de Calama muestran que la temperatura y humedad relativa, $\mathrm{NOx}$, $\mathrm{CO}$ y dióxido de azufre $\left(\mathrm{SO}_{2}\right)$ se correlacionan significativamente con consultas de urgencia respiratoria en niños de 5 a 14 años en servicios de Atención Primaria de Salud (SAPU, SAR) $y$ de emergencia hospitalaria del hospital Carlos Cisternas, de Calama $(2.400 \mathrm{msnm})^{7}$.

La zona vecina al complejo industrial Puchuncavi-Ventanas situada en la región de Valparaíso, concentra varias industrias químicas y ha sido escenario de múltiples emergencias ambientales por contaminantes aéreos. En el aire se ha detectado $\mathrm{SO}_{2}$, tolueno, metil-cloroformo y nitrobenceno. Las poblaciones afectadas han sido escolares, motivando consultas de emergencia en el hospital de Quintero por presentar mareos, vómitos, cefalea, irritación, síntomas neurológicos, con escasos síntomas respiratorios. Se ha detectado metales pesados y arsénico en el suelo y también en fanéreos (uñas y pelo). Aún no se conoce con precisión el/los agentes causales de estas emergencias médicas, contemplándose en un futuro cercano la creación de una primera unidad de Pediatría ambiental.

El funcionamiento de las Centrales Termoeléctricas de Carbón (CTEC) constituye un problema de salud pública por sus efectos en la salud de la población. Una investigación ecológica realizada en comunas de similares condiciones socio-económicas, evaluó egresos hospitalarios en poblaciones expuestas a la operación de CTEC (Tocopilla y Huasco), siendo Caldera la ciudad de referencia. Asma y bronquitis mostraron mayor frecuencia de consulta en las ciudades expuestas, hallazgo consistente con investigaciones similares ${ }^{8}$. Revisiones enfocadas a evaluar los efectos tempranos en la vida sugieren asociación con prematurez y alteraciones en la antropometría de recién nacidos ${ }^{9}$. Se han descrito también efectos diferidos en funciones cognitivas, en la esfera reproductiva y en enfermedades cardiovasculares asociadas a concentraciones elevadas de material particulado y CO.

Un estudio realizado en la región del Maule, ha identificado un fenotipo específico de la EPOC por combustión de biomasa, con predominio en mujeres, mayor frecuencia de sibilancias, con aumento de IgE, compromiso de vía aérea pequeña, asociada con áreas pulmonares parchadas en la radiología de tórax y con atrapamiento de aire, siendo frecuente la superposición de asma y EPOC. La bronscoscopía muestra en estos casos antracosis en vías aéreas. Se debe sospechar este fenotipo en exposiciones por más de 6 meses a humo de biomasa ${ }^{10}$.

El uso de propelentes en los fármacos inhaladores presurizados deja huella de carbono y aumentan el efecto invernadero. No obstante su mayor costo, los fármacos elaborados en base a cartuchos inhaladores de polvo seco debieran reemplazar a los inhaladores presurizados, por su menor impacto ambiental y buenos resultados terapéuticos. 
Otra faceta de la CA analizada fue la implementación del plan de descontaminación atmosférica de la comuna de Osorno (Decreto 47, marzo 2016). La monitorización de niveles de contaminación en esta ciudad parece no tener adecuada representación geográfica, detectándose diferencias zonales al utilizar medidores portátiles de material particulado. La contaminación es mayor en zonas socioeconómicamente más desposeídas (Río Rahue norte), probablemente por uso de leña más contaminante y de menor costo (húmeda y desechos de madera con pintura).

La exposición a las emanaciones de incendios forestales determina un problema creciente de salud pública en el mundo. Se describe asociación positiva entre la exposición a estas emanaciones con la mortalidad general y la morbilidad por asma, EPOC y neumonía. Sus efectos en personas mayores de 65 años conllevan aumento en consultas por enfermedades respiratorias agudas, exacerbación de enfermedades respiratorias crónicas e infarto agudo del miocardio. Son también susceptibles a estos episodios, enfermos respiratorios crónicos y posiblemente cardiovasculares, personas en edades extremas y embarazadas. Es plausible considerar que estos episodios puedan tener influencia en la incidencia de cáncer, lo que justifica considerar sistemas de vigilancia adecuados ${ }^{11,12}$.

En el año 2018, la Organización Mundial de la Salud estimaba en 7 millones de personas la mortalidad global anual por contaminación del aire. Los confinamientos determinados por la pandemia COVID19 disminuyen la concentración de material particulado, reportándose que el virus SARS-CoV-2 puede ser transportado por este material permaneciendo hasta 3 horas suspendido en el aire. La exposición a contaminantes, especialmente PM ${ }_{2,5}$ aumentan la susceptibilidad a SARS-CoV-2. La revisión sistemática de Katoto et al. ${ }^{13}$ infiere efectos agudos de contaminantes en la incidencia y mortalidad por COVID-19, en especial para $\mathrm{PM}_{2,5}$ luego $\mathrm{PM}_{10}, \mathrm{NO}_{2}$ y ozono (este último sólo para la incidencia). Para exposición crónica se reportan asociaciones similares para $\mathrm{PM}_{2,5}$ y $\mathrm{NO}_{2}$. No obstante, estos resultados preliminares deben interpretarse con cautela dadas las debilidades metodológica de los estudios primarios incluidos en esta revisión.

Las ponencias presentadas en estas jornadas entregaron una buena actualización del nivel de conocimiento sobre los efectos para la salud derivados de la CA. Como una muy buena señal de avance local en el tema tratado en estas jornadas, se destaca la presentación de investigaciones originales desarrolladas por los expositores en Chile o en colaboración internacional.

Chile debe disponer de planes de mitigación de la $C A$, que reconozcan las realidades locales. Los ciudadanos tienen derecho a saber de sus riesgos ambientales y los investigadores tienen la obligación de promover la comunicación del riesgo a la población expuesta.

Dr. Manuel Oyarzún G. ${ }^{1,2}$ y Dr. Gonzalo Valdivia C. ${ }^{1,3}$ ${ }^{1}$ Comisión de Contaminación Ambiental, Sociedad Chilena Enfermedades Respiratorias ${ }^{2}$ Profesor titular, ICBM, Facultad de Medicina, Universidad de Chile ${ }^{3}$ Profesor titular, Depto. Salud Pública, Pontificia Universidad Católica de Chile Emails:manueloyarzun@uchile.cl - valdivia@med.puc.cl

\section{Bibliografía}

1.- OYARZÚN M. Contaminación atmosférica de Santiago: Realidades y proyecciones futuras. Rev Chil Enferm Respir 1991; 7 (3): 183-5. (Editorial).

2.- SERA F, ARMSTRONG B, TOBIAS A, VICEDOCABRERA AM, ÅSTRÖM C, BELL ML, et al. How urban characteristics affect vulnerability to heat and cold: a multi-country analysis. Int J Epidemiol 2019; 48: 1101-12.

3.- LIU C, CHEN R, SERA F, VICEDO-CABRERA AM, GUO Y, TONG S, et al. Ambient particulate air pollution and daily mortality in 652 cities. N Engl J Med 2019; 22; 381 (8): 705-15.
4.- HYSTAD P, LARKIN A, RANGARAJAN S, ALHABIB KF, AVEZUM Á, CALIK KBT, et al Associations of outdoor fine particulate air pollution and cardiovascular disease in 157436 individuals from 21 high-income, middle-income, and low-income countries (PURE): a prospective cohort study. Lancet Planet Health 2020; 4 (6): e235-45.

5.- CAI Y, HANSELL AL, GRANELL R, BLANGIARDO M, ZOTTOLI M, FECHT D, et al Prenatal, early-life, and childhood exposure to air pollution and lung function: The ALSPAC Cohort. Am J Respir Crit Care Med 2020; 202 (1): 112-23.

6.- GUARNIERI M, BALMES JR. Outdoor air pollution and asthma. Lancet 2014; 383: 1581-92. 
7.- CARRILlO J, MUÑOZ F, SILVA I, IBACACHE G, NICOLIS O. Consultas de urgencia respiratoria y su asociación con factores ambientales en niños de 5-14 años en la comuna de Calama. Rev Chil Enferm Respir 2020; 36: S40 pág 42 (Resumen CL-11).

8.- VIVIANI P, LEIVA C, OJEDA MJ, AHUMADA E, CORTÉS S. Daños de salud respiratoria en comunas expuestas a centrales termoeléctricas a carbón en el norte de Chile: análisis de datos secundarios. Rev Chil Enferm Respir 2021; 37 (1): 17-25.

9.- CORTÉS AS, YOHANNESSEN VK, TELLERÍAS CL, AHUMADA PE. Exposición a contaminante provenientes de termoeléctricas a carbón y salud infantil: ¿Cuál es la evidencia internacional y nacional? Rev Chil Pediatr 2019; 90 (1): 102-14.

10.- OLLOQUEQUI J, JAIME S, PARRA V, CORNEJO-CÓRDOVA E, VALDIVIA G, AGUSTÍ À, et al.
Comparative analysis of COPD associated with tobacco smoking, biomass smoke exposure or both. Respir Res 2018; 19 (1): 13.

11.- SANDOVAL D, REYES T, OYARZÚN M. Mecanismos de los efectos nocivos para la salud de la contaminación atmosférica proveniente de los incendios forestales Rev Chil Enferm Respir 2019; 35 (1): 49-57.

12.- OYARZÚN M. Efectos para la salud de la contaminación atmosférica proveniente de los incendios forestales. Bol Academia Chilena de Medicina 2020; 57: 84-8.

13.- KATOTO PDMC, BRAND AS, BAKAN B, OBADIA PM, KUHANGANA C, KAYEMBE-KITENGE $\mathrm{T}$, et al. Acute and chronic exposure to air pollution in relation with incidence, prevalence, severity and mortality of COVID-19: a rapid systematic review. Environ Health 2021; 10; 20 (1): 41. 